\title{
Many-valued Logics for Reasoning: Essays in Honor of Lluís Godo on the Occasion of his 60th Birthday
}

\author{
Didier Dubois ${ }^{1} \cdot$ Francesc Esteva $^{2} \cdot$ Tommaso Flaminio $^{2} \cdot$ Carles Noguera $^{3} \cdot$ Henri Prade $^{4} \cdot$ Ricardo O. Rodríguez $^{5}$
}

Published online: 6 March 2019

(c) Springer-Verlag GmbH Germany, part of Springer Nature 2019

This special issue is dedicated to Lluís Godo in the occasion of his 60th anniversary. Lluís is a man with solid academic foundations. He studied Mathematics at the University of Barcelona and Industrial Engineering at the Technical University of Catalonia, and throughout his academic life, he has been loyal to both traditions: the purely scientific and the applied. Even though he has published numerous theoretical works, he never shied away from finding how to model and solve a practical problem.

Lluís enjoys a privileged intelligence, primed with a knack for recognizing significant ideas that he shares generously with his numerous $\mathrm{PhD}$ students, with everyone that collaborates with him and with anyone that attends one of his lectures. Lluís is an achiever: "he is always on", quipped someone who knows him well. And this is something that-

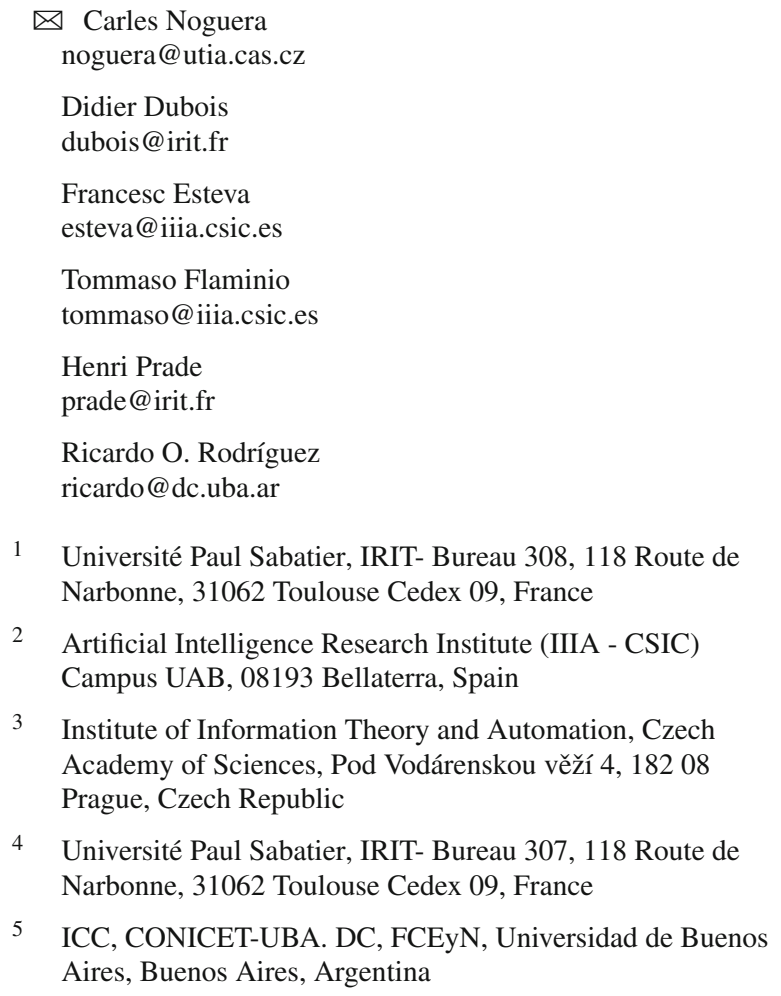

1 Université Paul Sabatier, IRIT- Bureau 308, 118 Route de Narbonne, 31062 Toulouse Cedex 09, France

2 Artificial Intelligence Research Institute (IIIA - CSIC) Campus UAB, 08193 Bellaterra, Spain

3 Institute of Information Theory and Automation, Czech Academy of Sciences, Pod Vodárenskou věží 4, 18208 Prague, Czech Republic

4 Université Paul Sabatier, IRIT- Bureau 307, 118 Route de Narbonne, 31062 Toulouse Cedex 09, France

5 ICC, CONICET-UBA. DC, FCEyN, Universidad de Buenos Aires, Buenos Aires, Argentina

as a prominent scholar once said-is earned only through hard continued effort nurtured with care. Moreover, Lluís also has an eager curiosity (a necessary, but not sufficient, condition to be a good researcher) that makes him probe into plenty of articles that fall into his hands. This involvement attracts researchers who know that he will look into their work and provide advice. No wonder he manages to produce so many of those reviews one dreams to get: reviews that not merely evaluate one's paper but reveal new bearings for one's own research. Naturally, these qualities have led not only to a bountiful publishing with co-authors of all kinds and topics, but also to earn Lluís a place in the Editorial Board of the best journals in the field. He is actually coEditor-in-Chief of Fuzzy Sets and Systems, Associate Editor of Artificial Intelligence Journal and Autonomous Agents and Multi-Agent Systems and member of the Editorial Board member of Progress in Artificial Intelligence. He is also fellow of ECAI and has been vice-President of EUSFLAT and ACIA; General Chair or program (area) chair of many conferences: program chair of FUZZ-IEEE 97 held in Barcelona (the first time that this conference was held outside USA) and several IJCAI, ECAI, UAI, ECSQARU, SUM, etc., conferences.

Lluís has deeply worked in many different fields. This fact is witnessed by the festschrift ${ }^{1}$ that has been published in occasion of his 60th anniversary where friends and colleagues have collected brief descriptions of Lluís' activity in the following areas: Argumentation Theory, Modal Fuzzy Logics for Uncertainty, Mathematical Fuzzy Logic, Possibility theory: logic, similarity and decision, Similarity reasoning, Paraconsistent Fuzzy Logic and Fuzzy Modal Logic. In all of these topics, Lluís has contributed with fruitful theoretical and applied ideas.

This special issue mainly contains extended versions of contributions presented to the international conference Many-Val 2017 that was held in Toulouse in November 2017

\footnotetext{
1 The volume can be downloaded at https://www.iiia.csic.es/ sierra/ wp-content/uploads/2019/02/Godo.pdf.
} 
and this is the reason why the papers of this volume mainly focus in many-valued logic, one of the topics where Lluís is best known. Indeed, Lluís was deeply involved in the creation of the discipline that nowadays goes under the name of Mathematical Fuzzy Logic and which was initially led by Petr Hájek in the early 90's. Hájek was an outstanding logician that promoted the interest of many researchers, including Lluís and Francesc Esteva, in the formal development of Fuzzy Set Theory. Zadeh himself called this approach "Fuzzy Logic (in a narrow sense)." In the area of Mathematical Fuzzy Logic, Lluís collaborated with many other well-known logicians. Let us cite first Franco Montagna a great logician and better person with whom Lluís maintained a close and fruitful relationship for many years. Lluís also collaborated with Roberto Cignoli and Siegfried Gottwald two of the most relevant logicians working in this discipline. Unfortunately, Petr, Franco, Roberto and Siegfried passed away and we do not have the opportunity to have papers of them in this special issue. Nevertheless, the present volume collects articles which provide an enjoyable and interesting insight on this vibrant area of knowledge to which Lluís has contributed so much in his prolific career.

In conclusion, we are honored to say that Lluís is a good person in the noblest sense of the word. Friend of his friends, he never has a "no" for an answer and you know you can always count on him. He is an exceptional, unique and unrepeatable human being.

Congratulations for the work done and for the anniversary!

This issue has gathered sixteen contributions in a variety of topics, all related to Lluís' research interests: ${ }^{2}$

(1) Stefano Aguzzoli and Matteo Bianchi present a study on varieties of MTL-algebras whose lattice of subvarieties is totally ordered. Such varieties are called linear and one of the main results of their paper shows that a variety $\mathbb{L}$ of MTL-algebras is linear if and only if each of its subvarieties is generated by one chain.

(2) The paper by Teresa Alsinet, Josep Argelich, Ramón Béjar, Joel Cemeli presents an experimental investigation on Twitter discussions. They exhibit an algorithm that scales at most with linear time with respect to the size of the discussion.

(3) Arnon Avron focuses on the need of infinite matrices for paraconsistent logics showing that most of the paraconsistent logics which have been investigated in the literature have no finite characteristic matrices and in the most important cases not even finite characteristic non-deterministic matrices.

\footnotetext{
2 The papers coauthored by any of the guest editors of this issue have been handled by a regular editor of the journal to ensure an unbiased reviewing and decision process.
}

(4) The paper by Guillermo Badia, Vicent Costa, Pilar Dellunde and Carles Noguera brings some new contributions to graded model theory (that is, the logical study of first-order fuzzy structures) by giving syntactical characterizations of classes of structures in terms of their axiomatizability by universal and universalexistential sentences.

(5) Miquel Bofill, Felip Manyà, Amanda Vidal and Mateu Villaret aim at finding challenging benchmarks for Łukasiewicz satisfiability solvers. Their paper starts by defining a natural and intuitive class of clausal forms (simple Ł-clausal forms) and studies their complexity. They prove that the satisfiability problem of simple $€$ clausal forms can be solved in linear time.

(6) The paper by Manuela Busaniche, Penélope Cordero and Ricardo Oscar Rodriguez introduces the class of Pseudomonadic BL-algebras with the aim of approaching an open problem proposed by Hájek in his book, i.e., to find an elegant axiomatization for the modal logic KD45 in the algebraic setting of BL-algebras.

(7) Carlos Caleiro, Sergio Marcelino and Joao Marcos investigate the combination of fragments of classical logic as a way of conservatively extending a given Boolean logic by the addition of new connectives. Their main result is a characterization of the conditions under which such a combination produces the corresponding fragment of classical logic over the signature containing connectives from both fragments.

(8) Petr Cintula, Paula Menchón and Carles Noguera introduce a new form of frame semantics for modal many-valued logics, by endowing lattice-valued neighborhood frames with admissible sets of worlds that restrict possible evaluations and allow to prove a very general completeness theorem.

(9) Marcelo Esteban Coniglio and Ana Claudia Golzio study, in their contribution, swap structures semantics for some Ivlev-like modal systems.

(10) Esther Anna Corsi and Christian Fermüller explore systematic connections between weighted (semi-abstract) argumentation frames and t-norm based fuzzy logics. To this aim, they introduce the concept of argumentative immunity, as well as corresponding notions of argumentative soundness and completeness with respect to given sets of logical attack principles.

(11) The paper by Rodolfo Ertola-Biraben concerns with meet-complemented lattices expanded with the greatest regular below, which is right adjoint of the double meet-complement. His main result shows that such is an equational class satisfying the Stone equality.

(12) The paper by Zuzana Haniková introduces a notion of implicit definability in the framework of propositional Łukasiewicz logic. Several variants of implicitly defining each of the rational elements in the standard 
semantics are explored, and based on that, a faithful interpretation of theories in Rational Pavelka logic in theories in Łukasiewicz logic is obtained.

(13) The paper by Daniele Mundici is about betting methods for continuous independent events. His main result shows that product is the only coherence preserving operation on books on logically independent sets of continuous events.

(14) The paper by Matthew Spinks, Umberto Rivieccio, Thiago Nascimento investigates the meaning of the Nelson identity in the context of compatibly involutive commutative integral residuated lattices and proves that a compatibly involutive commutative integral residuated lattice is a Nelson residuated lattice iff it is $(0,1)$-congruence orderable iff it is $(0,1)$-Fregean.

(15) The paper by Vicenç Torra and Yasuo Narukawa provides an extension of the use of game theory for network analysis by applying, to this context, Choquet and Sugeno integrals with respect to nonadditive measures.
(16) Diego Valota investigates in his contribution the logic RDP and he obtains a functional representation of RDP logic formulas. Further, he provides a combinatorial representation for finite RDP-algebras.

\section{Compliance with ethical standards}

Conflict of interest The authors declare that they have no conflict of interest.

Publisher's Note Springer Nature remains neutral with regard to jurisdictional claims in published maps and institutional affiliations. 